\title{
The Differences in Diagnosis between Western and Oriental Medicine
}

\author{
National Medical Center Hospital \\ Anesthetic Department

Kumio YAMASHITA
Misao TAKENOUCHI
Toshio YOGOSAWA
Mariko IGUCHI

The main points of difference between Western aud Oriental medicine are as follows :

1) Oriental medicine is concerned mainly with treatment methods and. Western medicine with the diagnosis of the disease.

2) Oriental medicine, in a word, is based on the superficial anatomy and Western on the pathological or microscopic.

3) Oriental medicine is more philosophical and Western more scientific.

4) Oriental medicine uses the feeling of our bodies and Western uses mechanical methods.

5) Oriental medicine is more deductive, and Western more analytical.

6) Oriental medicine is more functional and Western more constructive.

At any rate, I believe that Western medicine is more progressive and scientific, and yet we cannot really compare the two because they are, as it were, fighting in two completely different arenas.

Diagnosis of the patient is made by four methods :-

1) inspection, 2) auscultation, 3) anamnesis and 4) palpation.

Among palpation methods one of the most important is pulse diagnosis. For this diagnosis we usually feel $A$. radialis and $A$. carotis. When A.radialis is used, we feel that artery with the tips of the $2 \mathrm{nd}, 3 \mathrm{rd}$, and 4 th fingers and determine the condition of the artery from the respective points, and then give diagnosis of either fullness or dispersionof each of the 12 meridians. We notice Yang meridians on the superficial part and Yin meridians at a deeper level and call themsuperficial wave and deep wave respectively, and the pulse wave between them is called the medial pulse wave.

In one section they compare the width of the felt pulse on the radial artery and on the carotic artery and then diagnose the patient's condition as fullness or dispersion and determine what meridians will be the most important ones to be used in the treatment. (Jin-gei myakuha).

However, these pulse diagnosis methods are usually obscure and intensive training is necessary in order to make correct diagnosis. In some sections of acupuncture more than ten years' experience may be necessary before a doctor can attain a high standard of accuracy. This pulse diagnosis is more definite and objective when we use an electrical device. ${ }^{1)}$

An electrode of $6 \mathrm{~mm}$ in diameter and $2.5 \mathrm{~mm}$ in thickness has been deviced to record the pulse wave on the six original 
points on the patient's wrist. Pulsation is thought to be a wave made by the pressure of the blood against the walls of the arteries when it passes through from the heart to the peripheral arteries.

According to the indications on a pressure meter, the types of artery waves can be divided into two groups; one group is called fullness, the other is called empty. In the "fullness" group are floated wave, overflow or flooded wave, undulatory wave, full wave, strained wave, wet and damp wave, and deep wave. (fig1) In the "empty" group are delayed wave, slender wave, drop wave, and glassy wave. (fig 2)

Fig. 1

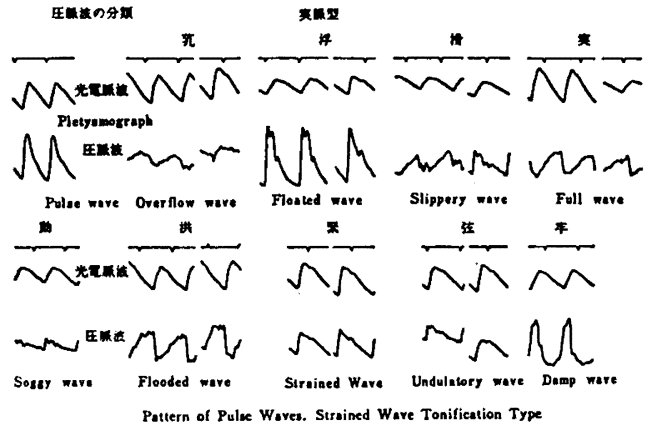

Fig. 2

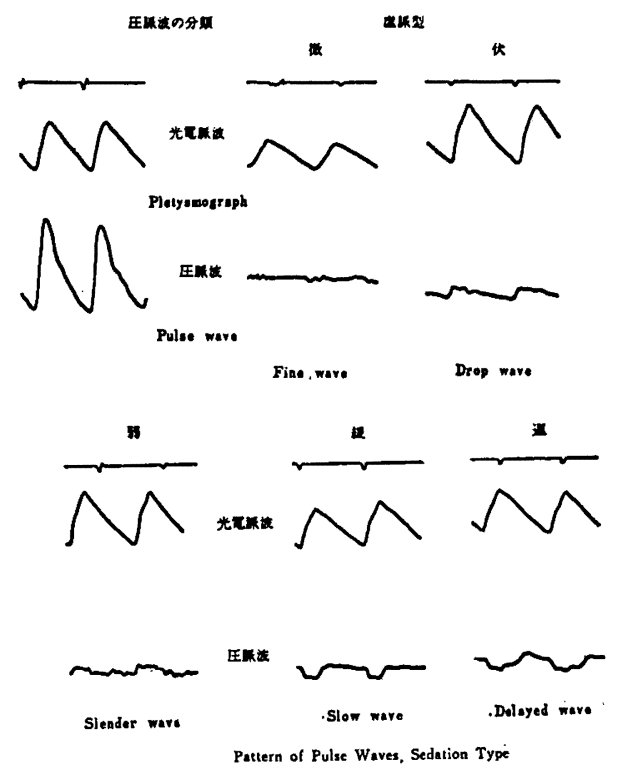

Dr. Akabane devised an excellent diagnosis method. The sensitivity to heat on the tip of the fingers, which are called Sei-ketsu (source points), is different according to fullness and emptiness of the respective meridians. The source points may be rubbed with a burning incense stick and the tolerance threshold is calculated by the number of times rubbing on that point can be endured. The more times that can be tolerated the fuller the meridian is which coincides with that point. Furthermore, the fullness may be treated by using acupuncture on the

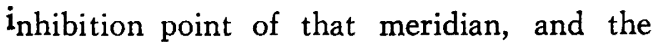
emptiness by acupuncture on the excitation

Fig. 3

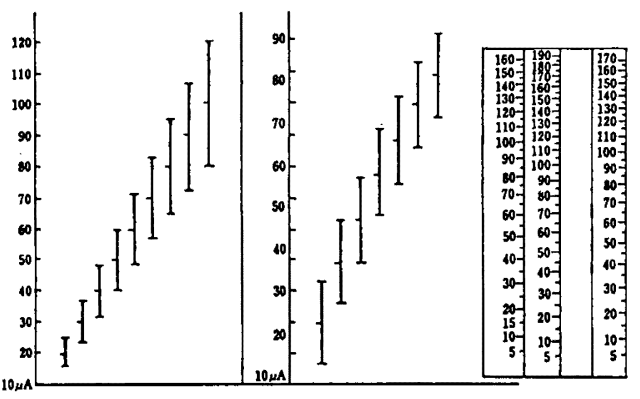

The determination of physiological range in Ryodoraku chan

Fig. 4

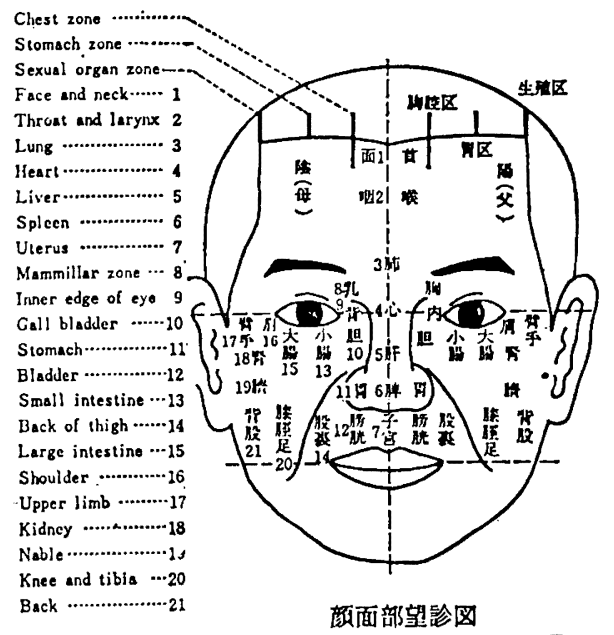

Figure of Colour Thermography on Face 
point. This method is called Akabane's method.

The Ryodoraku chart is device, a clear device, to determine the excitation and inhibition of all twelve meridians by measuring the electro-permeability on the measuring points on the patient's wrists and feet. This slide shows the representative points which most closely coincide with the so-called original points. ${ }^{2) 3}$ )

To decide the excitation and inhibition of a meridian it is important to determine the physiological or normal range. To get the physiological or normal range of the 24 meridians, firstly, the average value of the electric currents of all meridians must be calculated and then some width may be physiologically permitted to be normal may be added upwards and/or downwards from the average value. However, it may be said that the higher the current, the wider the width of the physiogical range.

As the figure 3 shows, the higher the average value, the wider the physiological range is. For example, when the average value is 100 microamperes, the physiological range is $2 \mathrm{~cm}$ and when the average value is 20 microamperes, the physiological range

Fig. 6

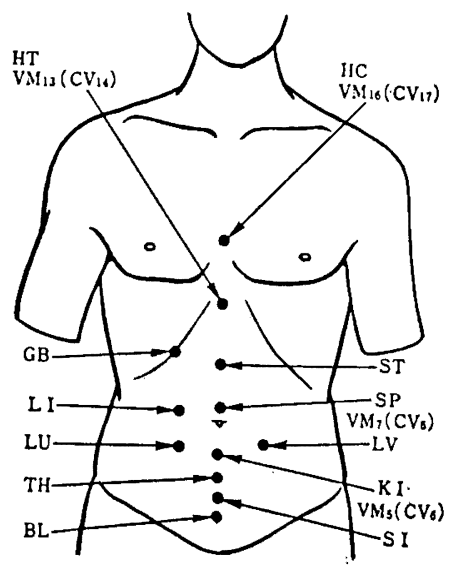

Pressure Diagnosis(Alarm Points) is $0.6 \mathrm{~cm}$. It is very inconvenient if the physiological range must be changed according to the average value of every meridian, so it was decided to have a $1.4 \mathrm{~cm}$ width for the physiological range when the average value is 50 microamperes. In so doing the range of the lower part of some meridians had to be extended and that of the upper part shortened. According to the result of this process the Ryodoraku chart was made. ${ }^{2}$ It was examined statistically later. ${ }^{4)}$ (fig 13 , 14)

Facial inspection is one of the inspection diagnostic methods in the field of acupuncture and moxibustion therapies. In the famous classic acupuncture book, "Rei-su", it is

Fig. 7

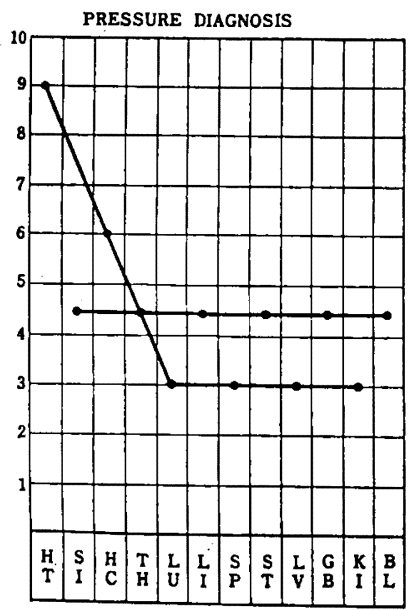

Fig. 8

LV $85 x$

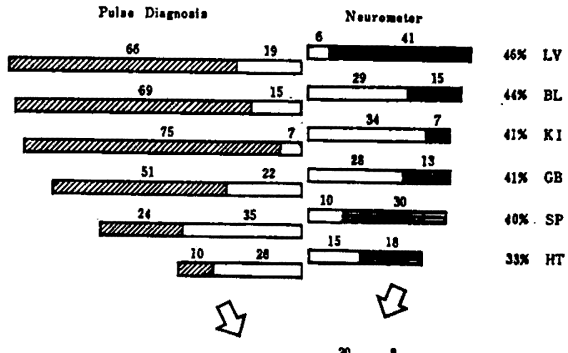

SI $16 x$

HT $14 \times$

LU $11 \times$

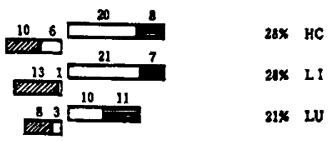


written that all energy and blood circulation of the 12 meridians and 365 longitudinal lines come up to the face. Therefore it can be said that all meridians concentrate in the face, which is divided into small areas each of which reveals the respective meridian phenomenon and/or organs and visceral functions. (fig 4) The temperature (cold, warm frozen and heated) the color, and the gloss of these parts of the face suggest the Yin and Yang, and the excitation and the inhibition of the meridians, and these features indicate which meridians can be treated effectively.

This inspection diagnostic methed up to this time has been done by subjective observation. However, the color thermography method is now used as a new inspection method in our clinic.

The faces of 36 volunteers where photographed by thermography and then the figures were analyzed and compared with the standard figure which is found in the classic

Fig. 9

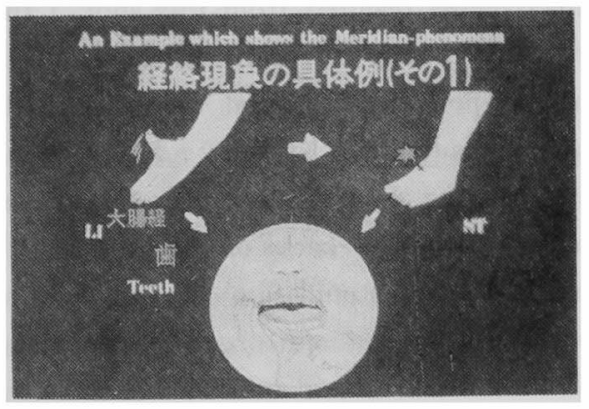

Fig. 10

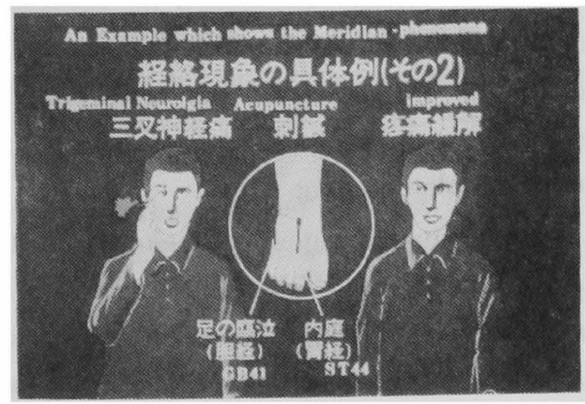

acupuncture book.

The black area in color thermography which shows the lowest temperature indicates inhibition, and the white, excitation. As the temperature is increased the color may change from black to blue, cyan, violet magenta, yellw, orange, red and white at the rise of every $0.3^{\circ} \mathrm{C}$. According to the color the degrees of excitation and inhibition may be divided as follows :

white and red means excitation ;

orange and yellow, mild excitation ;

magenta and green, medium (normal);

cyan and violet, mild inhibition ;

blue and black, inhibition. (fig 5 omitted)

Fig. 11

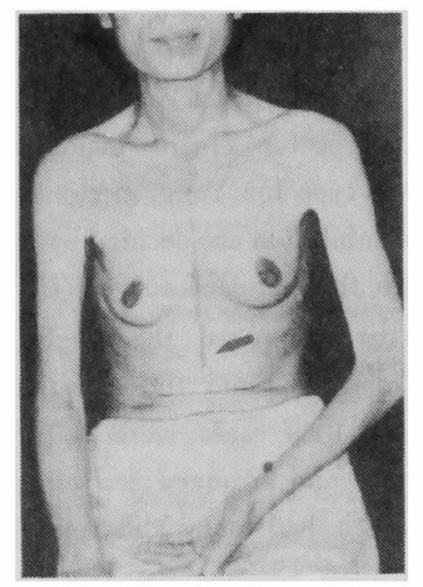

Fig. 12

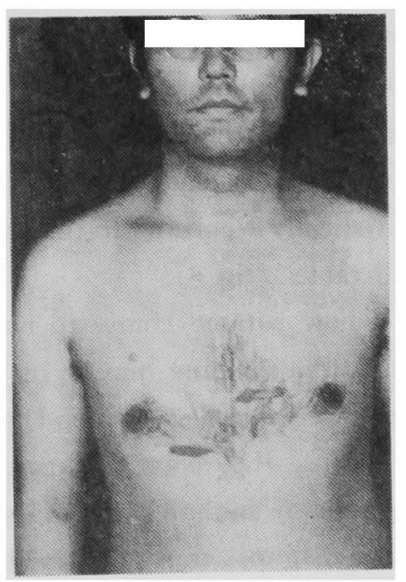


According to the results of thermography, we could surmise the possible complaints of the volunteers. We checked these complaints on our specially designed inspection diagnosis table and then compared them with the table on which the volunteers had marked their own complaints.

We gave marks from 0 to 100 depending upon how closely our results coincided with what the volunteers had written. If we consider a mark of over 51 as good, $80.5 \%$ showed good results. ${ }^{5)}$

In my clinic pressure diagnosis on the alarm points by using a specially designed tube is also used. When pressing on the representative alarm points with the tube, the intolerable pain thresholds are measured and recorded on the chart especially designed for this purpose. (fig 6) The standard curve shows L- type for Yin meridians and Japanese $1(-)$ type for Yang meridians. Excitation and inhibition are decided by comparing these with the standard curve. (fig 7)

When we compared the results of this pressure diagnosis and those of the Ryodoraku chart, 1000 cases were selected from both groups and arranged according to meridians which had many abnormalities. The liver meridian showed the largest number of abnormalities and the lung the least in both groups. It seems that the objective findings of pulse diagnosis and Ryodoraku diagnosis, by and large, show the opposite in excitation and the inhibition. However, these approaches seem to show the same value statistically according to the cumulative binominal table. (fig 8)

When a new patient comes to our clinic, we usually first examine him as in Western medicine ; for example, by blood tests, $x^{-}$ rays, CT-scans, etc.. Next we use the Oriental diagnosis method, say, Ryodoraku chart, pulse diagnosis and pressure diagnosis.
Then we decide what meridian lines and meridian points most important for his treatment. After 3 to 5 treatments, we decide whether this patient should continue to be treated by Oriental methods or change to Western methods and be sent to the specific departments according to his diseases. In my opinion, there are no clear definitive methods in Oriental medicine, and to get the correct diagnosis it is necessary to combine these Oriental methods with those of Western medicine for the sake of the PATIENT not the ILLNESS.

Here I would like to pick up several interesting cases from the viewpoint of Oriental medicine.

This patient had severe pain on the instep of his right foot when he spread the lst and 2nd fingers of his right hand, or when he wanted to put his right hand into his pocket. He had already visited many big university clinics but couldn't get any relief. I injected $5-10 \mathrm{ml}$ of Xylocaine into the spot where he complained of the most pain, but no effectiveness resulted from this shot. I then tried to explain his pain mechanism from the viewpoint of Oriental medicine in this way; spreading his lst and 2 nd fingers may stimulate the Large Intestine meridian and the pain on the back of his foot may be caused by the stimulation of the Stomach meridian. The common organ which belongs to both these meridians is the teeth, so toothache may be the source of the pain in his foot. When questioned he confessed he had a toothache, so we sent him to the dentist for treatment of his teeth and the severe pain on the back of his foot disappeared completely. (fig 9)

We have many trigeminal neuralgia patients in our clinic. Trigeminal neuralgia of the second branch may be easily alleviated by needle insertion at ST43 $\left(\mathrm{F}_{6} 3\right)$ and 
$\mathrm{GB} 43\left(\mathrm{~F}_{5} 2\right)$. This technique is very useful before we try nerve block because it relieves the facial pain which is sometimes so severe that even swabbing the face with alcohol for disinfection is almost unbearable. (fig 10)

This lady had severe chest pain after a cardiac operation for mitral valve displacement. She couldn't eat anything and had to be kept in bed for three weeks after the operation. Intercostal nerve blocks only gave temporary relief from pain. Then Naikan HC $6\left(\mathrm{H}_{2} 4\right)$ and Ekkaten (New point), the crosspoint of the lines which connect the two mammilla and the middle axillar lines, were acupunctured and the pain completely

Fig. 13

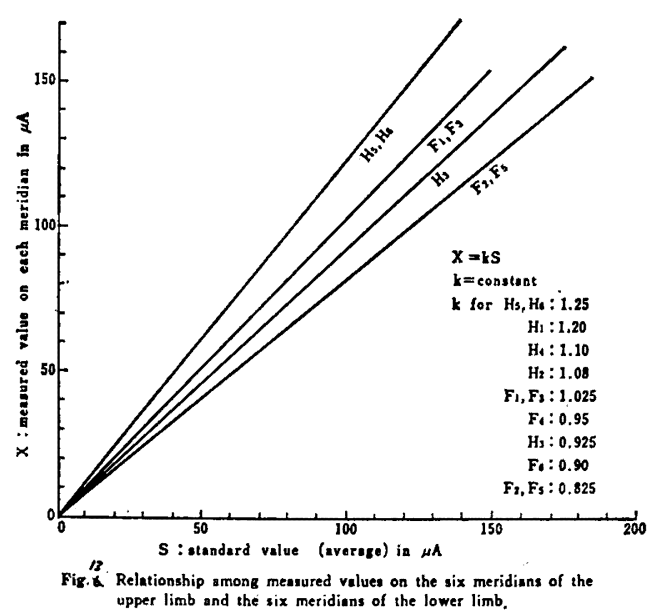

This Ryodoraku chart was statistically examined. An average of the 24 meridians of the body as the standard value, say $\mathrm{S}$ microamperes, may be set. When the measured value $\mathrm{X}$ microamperes on each meridian is compared, there are relatively constant relationships. For example, $\mathrm{X}$ on $\mathrm{H}_{1}$ is approximately 1.2 times the standard value (S), and $\mathrm{X}$ on $\mathrm{F}_{6}$ is 0.9 times $\mathrm{S}$. This relationship is expressed as a formula, $\mathrm{X}=\mathrm{kS}$, in which $\mathrm{X}$ is the measured microampere on each meridian and $\mathrm{k}$ the constant, calculated between 0.825 and 1.025. Their relationships are used in making a nomogram to compare different values on the same level. (Fig. 13) disappeared after two days' treatment. (fig 11)

After thymomectomy for myasthenia gravis, this man lost the sense of taste of his tongue almost completely. I thought that his taste disturbance may be related to the Heart and Heart Constrictor meridians so I tried acupuncture on the REPP on his scar in the sternal region and his sense of taste was considerably better after several treatments. (fig 12)

Of late acupuncture and moxibustion or herbal medicne have become popular in the world. Western medicine has its own disadvantages especially if it is used carelessly ;

Fig. 14

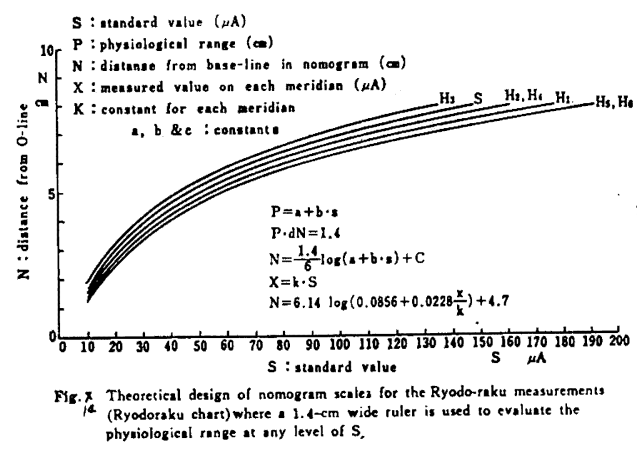

A total of $₫ 350$ healthy adults were chosen duri ng one year. They were selected without regard to seasons of the year, sex, etc. to determine th range lof measured values in healthy sub jects These $\mathrm{X}$ values are apparently unrelated to any pathological manifestations. This range, say $P$, seems to increase with the increase of $\mathrm{X}$ or $\mathrm{S}$. The relationship between $P$ and $S$ is expressed in the formula, $\mathrm{P}=\mathrm{a}+\mathrm{b} \cdot \mathrm{S}$, using the least square method. Here, $\mathrm{a}$ and $\mathrm{b}$ are constant and found to be 0.0856 and 0.0228 respectively (Colleration: 0.9946). (Fig. 14) In order to design a nomogram where $P$ is always scaled in the same width the higher parts of the scale must be gradually shortened. The Ryodoraku chart (a nomogram) was das designed empirically so that a ruler with a certain width can be used to show the physiological range at any level as mentioned above. 
however, I highly evaluate the splendid progress of Western medicine. As for Oriental medicine, its diagnostic methods may seem outmoded and unscientific in comparison to Western methods. Still, why has Oriental medicine gained so much interest and respect in recent years? The main reason may be that Oriental medicine concerns itself entirely with the patient himself while Western medicine is apt to focus only on the illness or the theory and often the patient is forgotten. I believe that Western medicine may be likened to a father's love, Oriental medicine to a mother's love ; neither one parent's love nor one school of medicine is complete in itself. The combination, Western-Oriental medicine, like God's love, is the ideal par excellence.

\section{References}

1) Yamashita, K., T. Yogosawa and M. Takenouchi : Methods to Reveal the Merid- ian Phenomenon Objectively, Journal of the Japan Acupuncture and Moxibustion Society, 27(2), 110, 1977

2) Nakatani, Y. and K. Yamashita : RYODORAKU ACUPUNCTURE, A Guide for the application of Ryodoraku, 44, Ryodoraku Research Instiute, Tokyo, 1977

3) Hyodo, M. : Ryodoraku Treatment, An Objective Approach Autonomic Nerve System Society, Osaka, 1975

4) Sato, T. a nd K. Nakatani : Acupuncture for Chronic Pain in Japan, Advances in Neurology, Volume 4, International Symposium on Pain, 813, Raven Press, New York, 1974

5) Takenouchi, M. and K. Yamashita : Inspectcon Diagnosis Method by Colour Thermography, Journal of the Japan Acupuncture and Moxibustion Society, 27(1) 122, 1977 